\title{
Role of Peer Relations and Family System on Academic Expectations Stress among Adolescents
}

\author{
Samera Jabeen ${ }^{1}$, Mamoona Ismail Loona ${ }^{2}$, Maryam Khurshid ${ }^{3}$
}

\section{Abstract}

The present study explored the role of peer relations and family system on academic expectations of adolescents. It was hypothesized that there would be a significant relationship among peer relations, family system and academic expectations of adolescents. A sample of 364 adolescents, boys $(n=139)$ and girls $(n=225)$ with the age range of 15-19 years was taken from different universities of Islamabad and Rawalpindi, Pakistan. Measures included Academic Expectations Stress Inventory by Ang and Huan (2006), and Index of Peer Relations by Hudson (1982), with use of correlational method. Results suggested significant correlation among study variables. A positive correlation of academic expectations was found with peer relations. Results showed nonsignificant difference in study variables with respect to gender. Regression analysis revealed that $23 \%$ variance was produced by peer relations in academic expectations. Results of One Way ANOVA showed significant difference between nuclear and joint family adolescents on peer relations, others expectations and self-expectations.

Key Words: Academic Expectations, Academic Expectations Stress Inventory, Peer Relations, Adolescents

Received: 22 November 2021; Revised Received: 20 December 2021; Accepted: 25 December 2021

${ }^{1} \mathrm{PhD}$ Scholar, Department of Psychology, International Islamic University, Islamabad, Pakistan.

${ }^{2}$ Assistant Professor, Department of Psychology, International Islamic University, Islamabad, Pakistan.

${ }^{3}$ Lecturer, Department of Psychology, International Islamic University, Islamabad, Pakistan.

\section{Corresponding Author Email:}

samera.mirza@gmail.com

\section{Introduction}

Families provide the bonding glue for the healthy and positive environment that encourages the adolescents to flourish their capabilities maximally. Studies prove that there are positive effects of family time and environment on development of children including school achievements as well as future career (Yeung \& Stafford, 2003). Research highlights the importance of the family system however, the impact of combined family system, which is the hallmark of the eastern culture has not been discovered vastly so far. Culture also regulate the family network by building family type, family size and form and the family functioning by defining barriers, rules, make connections between patterns, regulate practices, along with position and ranking in the family (McGoldrick et al., 2005). Adolescents of a nation, get in the colleges and universities with a motivation and clear purpose to achieve the goals that can secure a bright future for them. They also have to face many challenges during their struggle. Most of the psychotherapeutic intervention goals are set up for adults and needs of adolescents regarding the attainment of emotional demands for future concerns are neglected mostly. Hence, evidence from different

This article is distributed under the terms of the Creative Commons Attribution Non Commercial 4.0 License (http://www.creativecommons.org/licenses/by-nc/4.0/) which permits nonCommercial use, reproduction and distribution of the work without further permission provided the original work is attributed as specified. 
platforms suggest that prevention and treatment programs for adolescents are required (Das et al., 2016).

Adolescents of present age have to face not only the pressure of the expectations and demands of those related to them but also the hazards and difficulties which are a lot more intense than those faced by their elders during their time because traditionally Pakistan had joint family system and bonding within a family structure (Farooq et al., 2015). Adolescence is not the time of trouble and it also disagrees with excessive family and peer stressful situation during this time. On the other hand, adolescents see this time of transition as including challenges and trials that are related to physical, mental, and social development. Although this period of adolescence has certain advantages but there is another aspect to notice that today's adolescents are not given appropriate support and measures that can help them get through this difficult period in a smooth way into adulthood as a complete human being (Furstenberg et al., 2005). Apart from the stresses linked to bodily changes, adolescents have to go through another very important period during which they have to work up hard in excelling in academics so that they can get somewhere in the future.

Family system has a huge importance in developing basis of youngsters as they grow older and learn how to handle their life changes. One of the prominent functions of a family in eastern cultures is to determine career one has to pursue (Itrat et al., 2007). Having a successful academic life is very crucial for an adolescent's life as it becomes a benchmark about how successful a youngster would be in the future life such as university and practical life. Families and parents mold and shape the success of academic work of children as they can prove to be the great motivators for getting through tough academic testimonies. Based upon Parental Development Theory, a theory was derived that investigates the parental roles in the growing age of children. It also looks into the interaction ways of parents that may affect the well-being of a child (Mowder, 2005). The role of a parent can be modified and shaped by a number of reasons such as childhood experiences of parents and their background, culture, and society (Rosenberg et al., 1995).

The way children relate with their peers and friends, have an important play in determining their overall development mentally, physically as well as socially. Psychosocial adjustment in early childhood and adolescence can guarantee a balanced and healthy life ahead. It is very obvious and common for the teenagers to get intimate and close with their fellows as emotional maturity and socialization are interconnected so this maturity increases the relations with peers because during this time they are vulnerable naturally. There is a need for greater level of trust because of this vulnerability. Therefore, teen peer grouping and groups become very important during adolescence. It happens because it's the time when close friendships are formed and interpersonal relations are built due to this peer bonding. Before this age, only families are source of support and closeness but now, they become independent and relate with each other as an independent person. This greater interaction is in a way a symbol of independence among teens. Adolescents might choose to alter their choices, activities, speech, dressing, and behaviors to gain acceptance from their peers which is a source of feeling safe and belonged.

Adolescents respond to peer pressure when they modify their choice and behaviors to feel included by their friends. This conformity to peers can often result in adverse consequences such as wearing inappropriate clothes, skipping studies, using drugs or any other socially not approved activity. However, there can be positive influences of 
peer pressure too that most parents do not consider. If this association is positive and genuine, peers can influence each other in putting up hard work in studies, avoiding bad choices and making wiser choices regarding career choices as well because they are relatively mature cognitively as well as emotionally. As it is important for adolescents to "fit in" with friends so they might like to participate in the same activities and hobbies similar to their peers. They spend time with each other and share experiences to find a sense of belonging. Generally, youngsters will proceed toward their peers with whom they share common activities and interest, same cultural values, or a similar view regarding life. However, at times, it is possible for teens to get attracted to a very different group of individuals too. Peer groupings of adolescents are very different from the friendships of younger kids. These groups are more cohesive, bonded, dependent upon each other for feeling good. As the quality of relations change for teens due to their physical and mental changes, their friendships also get modified. They need more trust and closeness along with deeper commitments to feel protected and belonged. When adolescents have good friends around who stand by them and remain loyal, their confidence level also gets boosted (McLeod, 2013).

Although, adolescents enjoy good peer company but this bonding and closeness which is the hallmark of adolescent groups can also gravitate towards a number of issues in early years of adolescent life and later as well. Due to formation of certain groupings, some children definitely get left out and this social isolation and rejection can be painful especially for those kids who are sensitive. At other instances, negative labeling can also result due to certain activities, arguments, and interests of these adolescent groups. For example, movies and other social media has a major influence in portraying the typical image of popular and unpopular students which can lead towards tension and conflicts between different groups and can result in unpleasant consequences. Among these unpleasant consequences, bullying is one. It may happen when one group has strong power (mental, physical, social or financial) and control while other group is vulnerable and weaker (Patton, 2004).

The results of researches (Hussain et al., 2008) done on relationship between culture and self, explain how expectations give rise to stress related to academics among Asian students. Western culture has an independent concept of self while this concept is interdependent in Eastern culture. The concept of self is influenced by the orientation of culture so the culture itself will produce psychological differences that critical in forming the concept of the self of a person.

In another study, it was concluded that healthy relations, positive self-image, and self-respect has a positive impact upon expectations related to academics, career, future goals, exploring and planning careers. Bradley and Inglis (2012) have a stance that if students have suitable strategies and are able to use them actively, this may help them out in reducing the stress arising from any current social situation such as load of expectations that arise from family or peers. This might lead towards handling this situation mentally as well as physically in a positive way. Academic expectations get positively affected by the use of active and proper strategies to cope with the stress.

The major aim of present research is to explore the role of family system and peer relations on academic expectations among adolescents. The level of problems that hinder the academic performance among students is significant and it also leads towards problems related to psychological well-being of adolescents. Among these factors, support provided by parents in family 
and support provided by peers are basic source of influence for adolescents because these factors can impact the performance of adolescents. The expectations that arise from parents and teachers may also contribute in a student's perception of studies and success. Furthermore, the expectations a student set from self may also impact significantly the confidence level of a student in his/her academic capabilities.

All these factors add on to the difficulties of adolescents who are at a crucial stage of their lives where they are under a lot of pressure of making responsible decisions regarding career and future. The present research aims at knowing basic factors that can contribute towards the stress faced by adolescents with respect to how this stress can affect academic performance among Pakistani adolescents. Additionally, this study will be helpful in making an understanding of how the results can be beneficial in designing and implementing interventions for the adolescents, which can lead them towards a safer path in future. Further study of the factors that add on to difficulties of adolescents related to family, peer relations, and academic expectations of adolescents is desired.

\section{Method}

\section{Research Design}

Quantitative, cross sectional research design was used for current study.

\section{Sample}

Sample consisted of 364 adolescents as research participants $(N=364)$. The number of boys was $(n=139)$ while number of girls was $(n=225)$. All the participants were taken from different private and governmental institutes (colleges and universities) of Islamabad and Rawalpindi. The age range of participants was from 16 to 19 years, who belonged to different socioeconomic classes. Random sampling method was used to collect the data.
In the inclusion criteria, students of fresh study years were taken only. In the exclusion criteria, students repeating courses, or those taking the optional courses, or already passed students were not taken.

\section{Measures}

\section{Demographic Sheet and Consent Form}

To acquire some extra details about the participants, a demographic sheet was given along the scales. The sheet included information regarding name, age, gender, name of institute, family system, and monthly income. Moreover, informed consent form was also given to participants for taking their written consent to take part in the research. They were also insured of confidential information, protection from any kind of physical/emotional harm, right to withdrawal and right of privacy.

\section{Academic Expectations Stress Inventory} (AESI)

This scale measures the level of expectations as they can become a cause of stress. This scale was developed by Ang and Huan (2006) and it consists of nine items that measure the expectations on two aspects. One aspect is parents or teachers' expectations that consist of five items while the other is selfexpectations that consist of four items. On both aspects, participants have to rate their responses from 1 to 5 on each item. There are no cut-off scores or reverse coding. Minimum scores range is 9 while maximum score range is 45 for the scale. Scores on AESI can be calculated as total as well as subscale scores. Total scale Cronbach alpha value is (.89) and for self (.83), and for others (.87). This scale was used in English for data collection.

\section{The Index of Peer Relationship (IPR)}

The Index of Peer Relations (IPR) was developed by Hudson in (1982) is a scale which aims to measure severity and degree of some issue that peer members may encounter during their relationships with each other. It consists of 25 items that can be scored on a 
scale from 1 to 5 . Cut-off point for the scale is 30. Minimum scores range is 25 while maximum score range is 125 . Reveres scoring have been assigned to Items nos. 1, 2, $4,5,8,14,15,17,18,20,21$, and 23. Total scale Cronbach alpha value is (.90). This scale was used in English for data collection.

\section{Procedure}

A sample of 364 adolescents was taken for the current research as participants. All these adolescents were taken from different government and private universities of
Islamabad and Rawalpindi, with the help of random sampling method. Study participants were given the scales Academic Expectations Stress Inventory (AESI) and Index of Peer Relations (IPR) after they were briefed and taken informed consent. It was requested from participants to fill the scales as per their original opinions. For a smooth collection of data, institutional permission was also taken from concerned authorities. Moreover, the participants were insured of the confidentiality of their data.

\section{Results}

\section{Table 1}

Descriptive Statistics of Academic Expectation and Index of Peer Relations $(N=364)$

\begin{tabular}{|c|c|c|c|c|c|c|}
\hline \multirow[b]{2}{*}{ Scales } & \multirow[b]{2}{*}{ Mean } & \multirow[b]{2}{*}{ SD } & Score & Range & \multirow[b]{2}{*}{ Skew } & \multirow[b]{2}{*}{ Kurt } \\
\hline & & & Actual & Potential & & \\
\hline $\begin{array}{l}\text { Index of Peer } \\
\text { Relations }\end{array}$ & 55.00 & 16.28 & $22-99$ & $25-125$ & .40 & -.67 \\
\hline Others Expectations & 15.17 & 5.5 & $5-25$ & $5-25$ & .13 & .26 \\
\hline Self-Expectations & 12.60 & 4.31 & $4-20$ & $4-20$ & -.21 & -.83 \\
\hline
\end{tabular}

Note. Skew $=$ Skewness, Kurt $=$ Kurtosis

Table 1 shows the descriptive statistics for both variables used in the study. Mean scores for Index of Peer Relations is 55.00 and ranges from 22-99. Others Expectations is
15.17 and ranges from 5-25. Mean score of Self-Expectations is 12.60 and ranges from 420.

Table 2

Alpha Reliability of Index of Peer Relations (IPR), Others Expectations and Self-Expectations subscales $(N=364)$

\begin{tabular}{lll}
\hline Scales & No. of Items & $\boldsymbol{\alpha}$ \\
\hline Index of Peer Relations & 24 & .87 \\
Others Expectations & 5 & .81 \\
Self-Expectations & 4 & .78 \\
\hline
\end{tabular}

Note. $\alpha=$ reliability coefficient

Table 2 shows that the reliability for Index of Peer Relations scale is .87 , for Others Expectations subscale is .81 and for Self-
Expectations is .78 respectively. The alpha reliabilities of scales and subscales are acceptable and satisfactory. 
Table 3

Pearson Correlation of Index of Peer Relations (IPR) and Others Expectations and SelfExpectations Subscales

\begin{tabular}{lccc}
\hline Variables & $\mathbf{1}$ & $\mathbf{2}$ & $\mathbf{3}$ \\
\hline 1. Index of Peer relations & & $.39 * *$ & $.49 * *$ \\
2. Others Expectations & & & $.64 * *$ \\
3. Self-Expectations & & & \\
\hline
\end{tabular}

Note. $* * p<.01$

The Table 3 shows that there is strong significant positive relation between Index of Peer Relations Scale and Others Expectations and Self-Expectations subscales. Moreover, the above table showed that there is strong positive relation between index of peer relations and others expectations among adolescents. In the same way, the index of peer relations has positive relation with selfexpectations among adolescents.

\section{Table 4}

Linear Regression Analysis Showing Index of Peer Relations as Predictor of Academic Expectations among Adolescents $(N=364)$

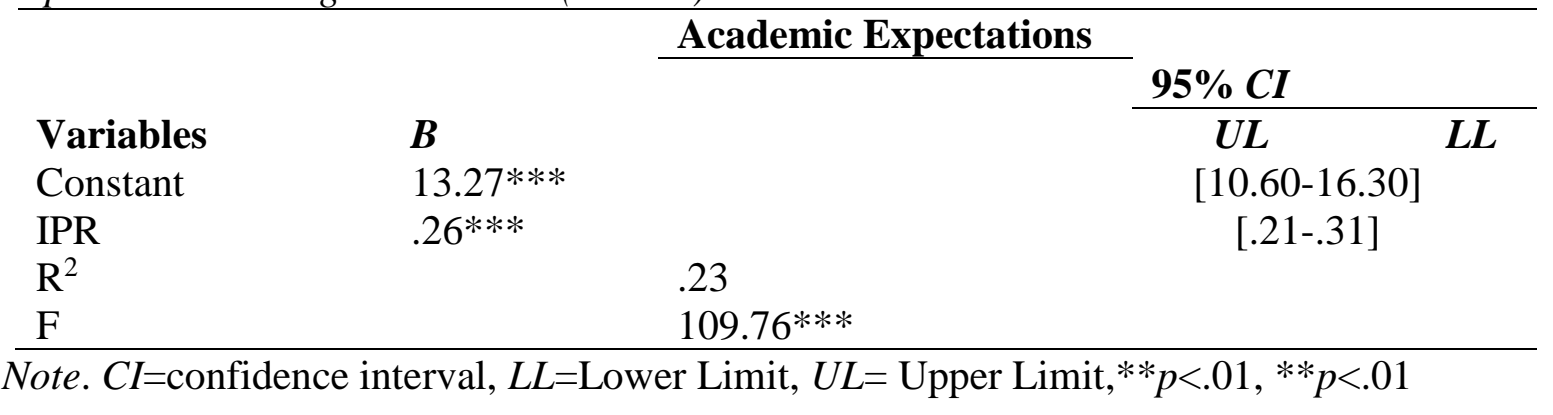

Table 4 shows that linear regression was used for this analysis. Table suggests that about $23 \%$ variance was produced by peer relations in academic expectations in the first step. In second step, academic expectations was added. Since the results were significant therefore, it can be concluded that peer relations is a significant predictor of academic expectations.

\section{Table 5}

Mean Differences in Family System on Index of Peer relations, Others Expectations and SelfExpectations $(N=364)$

\begin{tabular}{|c|c|c|c|c|c|c|c|c|c|}
\hline \multirow[t]{2}{*}{ Variables } & \multicolumn{2}{|c|}{$\begin{array}{r}\text { Nuclear } \\
(n=246)\end{array}$} & \multicolumn{2}{|c|}{$\begin{array}{l}\text { Joint } \\
(n=118)\end{array}$} & \multirow[b]{2}{*}{$t(362)$} & \multirow[b]{2}{*}{$p$} & \multirow{2}{*}{$\frac{95 \% \mathrm{CI}}{L L}$} & \multicolumn{2}{|r|}{ Cohen's d } \\
\hline & $M$ & $S D$ & $M$ & $S D$ & & & & $\boldsymbol{U L}$ & \\
\hline IPR & 54.70 & 16.65 & 55.67 & 15.50 & 9.67 & .000 & -18.94 & 12.53 & .05 \\
\hline $\begin{array}{l}\text { Others } \\
\text { Expectations }\end{array}$ & 14.83 & 5.54 & 15.89 & 5.27 & 5.26 & .000 & -4.28 & -1.95 & .19 \\
\hline $\begin{array}{l}\text { Self- } \\
\text { Expectations }\end{array}$ & 12.49 & 4.50 & 12.85 & 3.87 & 6.05 & .000 & -3.70 & -1.89 & .08 \\
\hline
\end{tabular}

Note. $p=$ significant value; $C I=$ confidence interval; $L L$ : lower limit; $M=$ mean; $S D=$ standard deviation. 
Table 5 shows that there is significant difference between nuclear and joint family adolescents on peer relations, others expectations and self-expectations. Further, the table shows that adolescents of joint family system have stronger peer relations as compared to adolescents of nuclear family system. The table shows that joint family adolescents have others expectations as compared to nuclear family adolescents. The above table also shows that there is a significant difference between adolescents of joint and nuclear families on selfexpectations.

\section{Table 6}

Mean Differences in Gender among on Index of Peer relations, Others Expectations and SelfExpectations $(N=364)$

\begin{tabular}{|c|c|c|c|c|c|c|c|}
\hline \multirow[t]{2}{*}{ Variables } & $\begin{array}{c}\text { Boys } \\
(n=139)\end{array}$ & $\begin{array}{c}\text { Girls } \\
(n=225)\end{array}$ & \multirow[b]{2}{*}{$t(362)$} & \multirow[b]{2}{*}{$p$} & \multicolumn{2}{|c|}{$95 \% C I$} & \multirow[t]{2}{*}{ Cohen's d } \\
\hline & $\mathrm{M} \pm \mathrm{SD}$ & $\mathbf{M} \pm \mathbf{S D}$ & & & $L L$ & $\boldsymbol{U L}$ & \\
\hline IPR & $54.97 \pm 15.10$ & $55.02 \pm 17.00$ & .03 & .977 & -3.51 & 3.41 & .00 \\
\hline $\begin{array}{l}\text { Others } \\
\text { Expectations }\end{array}$ & $15.14 \pm 5.05$ & $15.19 \pm 5.73$ & .08 & .933 & -1.21 & 1.11 & .00 \\
\hline $\begin{array}{l}\text { Self- } \\
\text { Expectations }\end{array}$ & $12.53 \pm 3.91$ & $12.66 \pm 4.56$ & .29 & .776 & -1.05 & .784 & .03 \\
\hline
\end{tabular}

Note. $p=$ significant value; $C I=$ confidence interval; $L L$ : lower limit; $M=$ mean; $S D=$ standard deviation.

Table 6 shows that there is non-significant difference between boys and girls on peer relations. The above table shows that there is non-significant difference between boys and girls students on others expectations. Further, the table shows that there is non-significant difference between boys and girls students on self-expectations.

\section{Table 7}

One Way ANOVA showing Monthly Income Differences (low, middle, high) on Index of Peer Relations, Others Expectations and Self-Expectations $(N=364)$

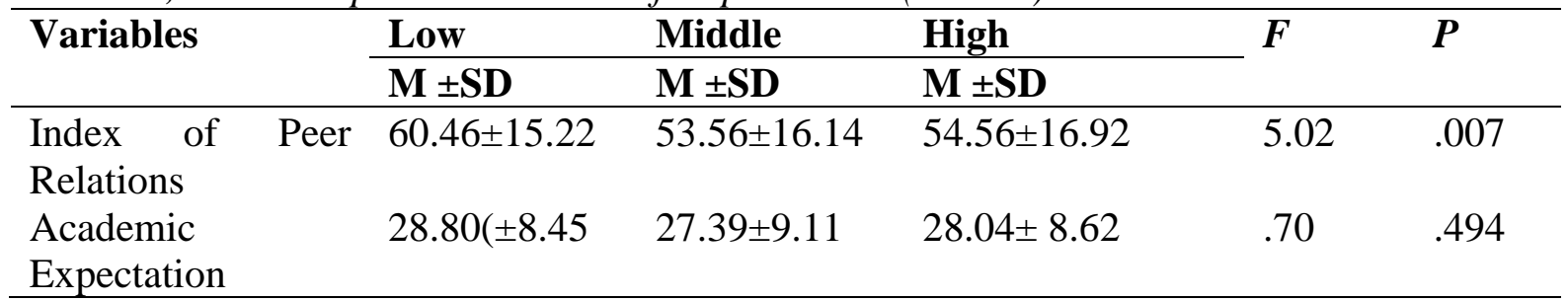

Note. $M=$ mean; $S D=$ Standard Deviation; $F=$ F-ratio; $p=$ significant value

Table 7 shows that there is non-significant difference on monthly income (low, middle, and high) on index of peer relations and academic expectation. However, multiple

\section{Discussion}

The current study was conducted to see the role of family and peer relations in academic analysis of Post Hoc showed that there is significant difference between low and middle income groups on index of peer relations.

expectations stress among adolescents. First hypothesis of the current study was that there will be significant positive relationship 
between peer relations and academic expectations. To determine this relationship, Pearson Product Moment Correlation was used and results supported this claim with a significantly positive finding. There are many researches that support current finding. Ang \& Huan (2006) were of the view that pressure (parental, peers, teachers) and stress can even lead students towards suicidal ideations, if it's adverse. Pike (2006) also explored this relationship and found that there is a significant positive relationship among stress and pressure, academic achievement and academic expectations. A number of reasons can support this claim such as teachers and parents expect a lot from children which results in high expectations in academics. Another reason can be that in collectivistic cultures, it's very common to expect a lot from children and Pakistan is basically a country with most of collectivistic culture environment so, the results are coherent with observed trends of our society. The second hypothesis of the current study was that academic expectations will be predicted by peer relations among adolescents. To check this assumption based upon hypothesis, linear regression was used. The results suggested that about 23\% variance was produced in index of peer relations by academic expectations in the first step. In second step, academic expectations was added. Since the results were significant therefore, it can be concluded that index of peer relations is a significant predictor of academic expectations. Among other studies that support the findings, Pike (2006) found that stress can predict academic expectation among intermediate students, this stress comes from factors such as pressure of performing well, that is maintained by self, peers, teachers and parents.

In third hypothesis of this study, it was hypothesized that there will be a significant difference in peer relations and academic expectation among adolescents from nuclear and joint families. To check this assumption, Independent sample t-test was used. The results of this analysis showed that there is a significant difference between adolescents of nuclear and joint families on peer relations and academic expectations. Therefore, this hypothesis was also accepted. To support this finding, a study is quoted which was done by Diener et al. (1995) and it came up with a conclusion that students from Asian and Asian American have more academic expectations and related stress which is maintained by peers and parents because of the reason that Asian culture is collectivistic in nature where extended family is also viewed as immediate family. As Pakistan is part of Asian culture, we also follow trends of collectivistic culture so; it's very obvious for our peers and parents to expect a lot from adolescents and unknowingly putting a lot of unwanted pressure upon a student as well.

In forth hypothesis of this study, it was hypothesized that there will be significant difference in peer relations and academic expectations among boys and girls. Once again, independent sample $t$-test was used to check this hypothesis. The results showed a non-significant difference between boys and girls on peer relations and academic expectations. Therefore, forth hypothesis was rejected. This non-significant result can be supported by a finding done by researchers Kausar and Munir (2004) that explains that in Pakistani culture, more academic expectations and pressure is bound to sciences students no matter they are boys or girls. Demographics are always a good source to gain an extra insight into study variables. Not only it's useful extra information, it can be used later on as well. One Way ANOVA was used upon monthly income groups (low, middle, high). The results of this analysis showed a nonsignificant difference upon low, middle and high income groups. So, this hypothesis was rejected. Bradley and Inglis (2012) also came 
up with similar results that a non-significant difference exists between stress and pressure (peers, parents) and how this stress and pressure is handled on basis of class.

\section{Implications of the Study}

Findings of this study may have some vital implications. First of all, there is a need for more information to deal with changing trends of current age so that today's adolescents can be equipped with all the necessary skills to deal with stress and pressures that impact their potential and ability to perform and excel. Additionally, these findings can be a great deal of help in designing and implementing the interventions that can bring good results and can save mental health of adolescents. Moreover, these results can be used in educating peers, teachers, as well as parents in developing more realistic and rational expectations from adolescents.

\section{Limitations and Suggestions}

Followings are the limitations and suggestions of the current study. In the current study, only peer relations and family system were seen with reference to academic expectations but there are many others factors which also contribute towards these expectations which were not covered. Only adolescents were taken as sample and all other students were ignored who are under similar expectations and pressures. No comparisons of major study areas was done. As it was a mini research project so only two measures were used. A major project must be done on the same variables for detailed and better findings. Data must be collected from different cities of Pakistan so that a better understanding can be reached regarding situation and facts. Indigenous measures should be used for data collection as they can represent our society better.

\section{Conflict of Interest}

The authors declared no conflict of interest.

\section{Source of Funding}

The authors stated no source of funding.

\section{References}

Ang, R. P., \& Huan, V. S. (2006). Academic Expectations Stress Inventory: development, factor analysis, reliability and validity. Educational and Psychological Measurement, 66, 522-539.

Bradley, G. L., \& Inglis, B. C. (2012). Adolescent leisure dimensions, psychosocial adjustment, and gender effects. Journal

of adolescence, 35(5), 1167-1176. https://doi.org/10.1016/j.adolescence .2012.03.006

Das, J. K., Salam, R. A., Lassi, Z. S., Khan, M. N., Mahmood, W., Patel, V., \& Bhutta, Z. A. (2016). Interventions for Adolescent Mental Health: An Overview of Systematic Reviews. The Journal of adolescent health: official publication of the Society for Adolescent Medicine, 59(4S), S49-S60. https://doi.org/10.1016/j.jadohealth.2 016.06.020

Diener, E., Suh, E. M., Lucas, R. E., Smith, H. L., Shao, B. P., Grant, G., \& Whittell, B. (1995). Subjective wellbeing: Three decades of progress. Psychological Bulletin, 125, 276-302

Farooq, A., Kayani, A. K., \& Ahmad, K. (2015). Marriage and family structures in the rural Punjab a shift from conservative to contemporary patterns. International Journal of Social Policy, 35(56),30624.

Furstenberg, F., Rumbaut, R. G., \& Settersten, R. A. (2005). On the frontier of adulthood: An introduction. Chicago: University of Chicago Press. 
Hudson, W. (1982). The clinical measurement package. A field Manual. The Dorsey Press. Homewood Illinois.

Hussain, A., Kumar, A., \& Husain, A. (2008). Academic stress and adjustment among high school students. Journal of the Indian Academy of Applied Psychology, 34, 70-73.

Itrat A, Taqui AM, Qazi F, Qidwai W. (2007). Family systems: perceptions of elderly patients and their attendants presenting at a university hospital in Karachi. Journal of Pakistan Medical Association, 57(2), 106-10.

Kausar, R., \& Munir, R. (2004). Pakistani adolescents' coping with stress: effect of loss of a parent and gender of adolescents. Journal 599-610. adolescence, 27(6), of https://doi.org/10.1016/j.adolescence .2003.11.015

McGoldrick M, Giordano J, Garcia PN. (2005). Ethnicity and family therapy. 3rd ed. New York: Guilford Press

Mcleod, J. (2013). An Introduction to Counselling (5th edition). Berkshire: Open University.
Mowder, B. (2005). Parent development theory: Understanding parents, parenting perceptions and parenting behaviors. Journal of Early Childhood and Infant Psychology, 1, 46-64.

Patton,W. (2004). The impact of value discrepancies and cultural identity on psychological and socio-cultural adjustment of sojourners. International Journal of Intercultural Relations, 15, 209-225.

Pike, W. (2006). Educational planning and academic achievement of middle school students: A racial and cultural comparison. Journal of Counseling \& Development, 73(5), 518-526.

Rosenberg, M. Schooler, C., Schoenbach, C., Rosenberg, F. (1995). Global selfesteem and specific selfesteem: Different concepts, different outcomes. American Sociological Review, 60, 141-156.

Yeung, W. J., \& Stafford, F. (2003). Intrafamily Child Care Time Allocation: Stalled Revolution or Road to Equality? http:// depts.washington.edu/crfam/seminar series02- 03/Yeung.doc 